\title{
Linear Systems and Discontinuous Dynamics
}

\author{
J. M. Schumacher \\ CWI, Amsterdam, The Netherlands, and \\ Tilburg University, Tilburg, The Netherlands
}

Dedicated to Paul Fuhrmann at the occasion of his sixtieth birthday

\begin{abstract}
A dynamical system may be called discontinuous if it is subject to abrupt changes in its dynamic characteristics. Such changes may be induced either externally ("time events") or internally ("state events"). Here we discuss so-called linear complementarity systems. These are piecewise linear systems in which state events occur due to transitions from one branch of an ideal-diode-type characteristic to the other. We present a precise definition of the dynamics of such systems and give sufficient conditions for well-posedness.
\end{abstract}

\section{Introduction}

Recent years have seen a growing interest in systems that are subject to abrupt changes in their dynamic characteristics. There are actually several different motivations for this interest, coming from various domains of science. A discontinuous dynamical system arises for instance when a continuous system is coupled to a controller that switches between a finite number of operating modes (for instance on and off); the switching may be induced by external inputs or by measurements taken from the system itself. A second source of discontinuous dynamics lies in processes that are subject to unilateral constraints; think for instance of a robot arm that comes into contact with a rigid surface, or a tank in a chemical plant that at some point becomes completely filled. Sudden changes are sometimes inherent in the physics of a situation; examples could be a fluid that starts to boil, or a crane that overreaches. In quite another context, one may be interested in computing or communication devices whose main purpose is to switch between discrete states but which are nevertheless subject to disturbances of a continuous nature, such as clock drift. Impetus for the study of discontinuous dynamical systems therefore comes both from control theory and from computer science, as well as from the modeling and simulation of mechanical and electrical systems and of chemical processes. 
Of course the different backgrounds of researchers in discontinuous dynamical systems give rise to different angles from which the field is studied. For those whose background is in differential equations, the main modeling alternative is to use a traditional smooth dynamical model. An important reason not to choose alternative can for instance lie in speed of simulation; certain phenomena that happen on a timescale much faster than the timescale of interest are then described as instantaneous transitions in an attempt to avoid time-consuming calculations. Fast (real-time) simulation of complicated physical systems is crucial for instance in the training of pilots but also for testing of software that interacts with a physical environment. If this is taken as a motivation to study discontinuous dynamical systems, then naturally aspects related to simulation are of prime interest. For those whose background is in computer science, the main modeling alternative is to use a traditional completely discrete model such as a transition graph, in which time does not appear at all. Such researchers will be motivated to introduce continuous time by applications in which time is for some reason critical; hence they will tend to be interested in verification aspects. It remains to be seen whether the field of discontinuous dynamical systems with its disparate approaches will evolve into a coherent area of study; for the moment however, the possibility of combining ideas from various directions certainly adds to the attractiveness of the subject.

There should be no suggestion that the study of discontinuous dynamical systems is new; in fact the history of the subject goes back at least several decades. One should mention the fundamental work of Filippov [5], the work that has been done on bang-bang control $[3,14]$ and on systems with relays or bistable elements $[18, \S 22]$, and the classical treatments of mechanical systems with unilateral constraints [17, 12]; all of this refers to the fifties and sixties. The term hybrid, which is currently often used for systems that incorporate both continuous dynamics and discrete switching, appears to have been employed in this way for the first time by Witsenhausen in 1966 [22]. The resurgence of interest in hybrid systems, as indicated for instance in [2], can be attributed to several factors. Among those are the fact that computer scientists are extending their interest from the computer itself to its environment, and the fact that control theorists are more and more aware that in many industrial applications the continuous format that has long been standard in control theory is not quite appropriate. Actually it is somewhat embarrassing for control theorists to note the discrepancy between the high state of development of continuous control theory and the relatively small amount of theory that is available on switching control, despite the fact that programmable logic controllers dominate the market in many corners of industry.

The above doesn't imply that the systematic study that has been made of continuous control systems in the past decades is irrelevant. On the contrary, it will be argued in this paper that, at least for some types of discontinuous dynamical systems, the availability of an extensive theory of continuous multivariable systems $[11,23,6,16,13]$ will be of crucial importance in advancing the theory beyond the 
stage that was reached in the fifties and sixties. The reasoning behind this may briefly be explained as follows.

In order not to complicate the discussion unnecessarily, let us consider 'closed' dynamical systems, i. e. systems with no inputs. (We will see that, despite appearances, control theory is of relevance to such systems.) In a closed hybrid system, switching will typically take place when the continuous variables in the system reach certain threshold values. In a number of cases, it is possible to describe the switching by a so-called complementarity characteristic. Such a characteristic involves two continuous variables, say $u(t)$ and $y(t)$, that are both required to be nonnegative; there are two associated operating modes, one in which $u(t)$ is constantly zero, and one in which $y(t)$ is constantly zero. If in the first mode the variable $y(t)$ reaches zero and tends to become negative, then a switch to the second mode occurs; and if in the second mode $u(t)$ tends to cross zero then the first mode becomes operative. If all the switching in the system can be described in this way, then we effectively have a continuous dynamical systems with a number of pairs of continuous variables $\left(u_{i}, y_{i}\right)$ that represent canonical switching elements. Because the switching elements are canonical, all properties of the closed system as a whole (existence and uniqueness of solutions, stability, etc.) must be expressible in terms of the continuous part with its associated variables $y_{i}$ and $u_{i}$, and this is where the input/output systems theory comes in.

A simple example will be given below in which the complementarity structure described in general terms above does indeed arise naturally. Actually the reader may already have noted that what was described is essentially the ideal diode characteristic, with current through and voltage across the diode as the complementary variables. The example below is mechanical and uses position and force as complementary variables. Another obvious application is in hydraulic systems with valves for which current and pressure may be taken as complementary. There are also less obvious applications. For instance, it has been shown that the mode switching brought about by any piecewise linear element can be described in complementarity terms [21]. The fact that such a canonical description is available may make piecewise linear modeling an attractive alternative to fully nonlinear modeling; in turn this would open large new grounds for discontinuous dynamical systems.

In this paper, the following conventions will be in force. For a positive integer $k, \bar{k}$ denotes the set $\{1,2, \ldots, k\}$. For $I \subset \bar{k}, I^{c}$ denotes the complementary set $\bar{k} \backslash I$. Given $M \in \mathbb{R}^{k \times l}$ and two subsets $I \subset \bar{k}$ and $J \subset \bar{l}$, the $(I, J)$-submatrix of $M$ is defined as $M_{I J}:=\left(m_{i j}\right)_{i \in I, j \in J}$. Following a convention in [4], we shall also write $M_{I}$. instead of $M_{I \bar{l}}$ and $M_{\bullet J}$ instead of $M_{\bar{k} J}$. For $a \in \mathbb{R}^{k}$ and $I \subset \bar{k}$, we write $a_{I}=\left(a_{i}\right)_{i \in I}$. A vector $a \in \mathbb{R}^{k}$ is called nonnegative, and we write $a \geqslant 0$, if $a_{i} \geqslant 0$ for all $i \in \bar{k}$. A (finite or infinite) sequence of real numbers is said to be lexicographically nonnegative if either it is a sequence of zeros or its first nonzero element is positive; we write $\left(a^{1}, a^{2}, \ldots\right) \succeq 0$. If $\left(a^{1}, a^{2}, \ldots\right)$ is a sequence of vectors, we write $\left(a^{1}, a^{2}, \ldots\right) \succeq 0$ when $\left(a_{i}^{1}, a_{i}^{2}, \ldots\right) \succeq 0$ for all $i$. 


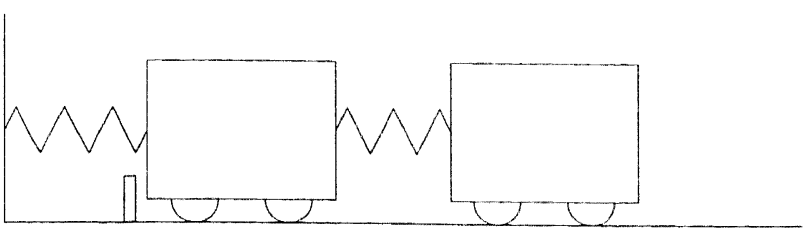

Figure 1: A discontinuous dynamical system.

\section{An example}

To motivate the development below, let us consider the following very simple example of a discontinuous dynamical system (see Fig. 1). Two carts are connected to each other and to a fixed wall by springs. The motion of the left cart is restricted by a purely non-elastic stop. For simplicity, we shall normalize all constants to 1 and let the springs be linear, and we shall assume that the stop is placed at the equilibrium position of the left cart. An 'event' takes place when the left cart hits the stop or when it is pulled away from a position at the stop. When an event takes place, the system switches from 'constrained mode' to 'unconstrained mode' or vice versa; these two modes may also be viewed as the two discrete states of the system.

Let us now discuss the dynamics of the system in the example. It is not difficult to write equations of motion for each of the two modes separately. Let $x_{1}(t)$ and $x_{2}(t)$ represent the deviations of the left and the right cart respectively from their equilibrium positions, and let $x_{3}(t)$ and $x_{4}(t)$ denote the corresponding velocities. In the unconstrained mode the equations (in first-order form) are the ones that would hold if there were no block:

$$
\begin{aligned}
& \dot{x}_{1}(t)=x_{3}(t) \\
& \dot{x}_{2}(t)=x_{4}(t) \\
& \dot{x}_{3}(t)=-2 x_{1}(t)+x_{2}(t) \\
& \dot{x}_{4}(t)=x_{1}(t)-x_{2}(t) .
\end{aligned}
$$

The equations of motion in the constrained mode are the ones that would hold if the first cart were nailed to the block:

$$
\begin{aligned}
& x_{1}(t)=0 \\
& \dot{x}_{2}(t)=x_{4}(t) \\
& x_{3}(t)=0 \\
& \dot{x}_{4}(t)=-x_{2}(t) .
\end{aligned}
$$

To give a complete description of the hybrid system, one also needs to specify under what conditions events take place and what the effects of such events will be. Under the assumption of inelastic collision, one can argue that a transition from the unconstrained mode to the constrained mode will occur at times $t_{0}$ when 
the following Boolean expression in terms of equality and inequality conditions on the state variables evaluates to TRUE:

$$
\begin{aligned}
& \left(x_{1}\left(t_{0}\right)=0\right) \wedge\left(( x _ { 3 } ( t _ { 0 } ) < 0 ) \vee \left(\left(\left(x_{3}\left(t_{0}\right)=0\right) \wedge\right.\right.\right. \\
& \left.\left.\left.\wedge\left(\left(x_{2}\left(t_{0}\right)<0\right) \vee\left(\left(x_{2}\left(t_{0}\right)=0\right) \wedge\left(x_{4}\left(t_{0}\right)<0\right)\right)\right)\right)\right)\right) .
\end{aligned}
$$

Moreover, when this event takes place the variable $x_{3}$ is reset to zero, whereas the other continuous state variables keep the values that they had just before the event. A transition from the constrained mode to the unconstrained mode will take place at times $t_{0}$ when the following expression evaluates to TRUE:

$$
\left(x_{2}\left(t_{0}\right)=0\right) \wedge\left(x_{4}\left(t_{0}\right)>0\right) .
$$

This event produces no jumps in the continuous state variables. Note that it is possible that the conditions for a transition from the constrained mode to the unconstrained mode are satisfied immediately after a transition from the unconstrained mode to the constrained mode has taken place; in such cases there are two events in one time instant (in a well-defined order).

The example shows that the specification of a hybrid system in the form of a "flat" differential automaton is not always very convenient. It is easier to specify the system in the form

$$
\begin{aligned}
\dot{x}_{1}(t) & =x_{3}(t) \\
\dot{x}_{2}(t) & =x_{4}(t) \\
\dot{x}_{3}(t) & =-2 x_{1}(t)+x_{2}(t)+u(t) \\
\dot{x}_{4}(t) & =x_{1}(t)-x_{2}(t) \\
y(t) & =x_{1}(t) \\
y(t) \geqslant 0, & u(t) \geqslant 0, \quad y(t) u(t)=0
\end{aligned}
$$

in which a constraint force denoted by $u(t)$ has been introduced. Note that the systems obtained by setting $u(t)=0$ and $y(t)=0$ in the above are indeed equivalent to (1) and (2) respectively; in the second case, note that imposing $y=0$ forces the relation $u=-x_{2}$ so that $u$ is an output even though the equations are written as if it was an input. Moreover, the transition condition from constrained to unconstrained mode is exactly the one that is needed to prevent $u(t)$ from becoming negative. The above system is an example of a linear complementarity system. A precise definition of this class of dynamical systems will be given in the next section. 


\section{Linear complementarity systems}

Consider the following system of linear differential and algebraic equations and inequalities:

$$
\begin{gathered}
\dot{x}(t)=A x(t)+B u(t) \\
y(t)=C x(t)+D u(t) \\
y(t) \geqslant 0, \quad u(t) \geqslant 0, \quad y^{\top}(t) u(t)=0 .
\end{gathered}
$$

The equations (9a) and (9b) constitute a linear system in state space form; we let the number of inputs be equal to the number of outputs. The relations (9c) are called complementarity conditions. Because of the nonnegativity constraints, the vanishing of the inner product $y^{\top}(t) u(t)$ implies that actually for each index $i$ at least one of the variables $y_{i}(t)$ and $u_{i}(t)$ must be zero. The set of indices for which $y_{i}(t)=0$ (we shall call this the active index set) need not be constant in time, so that the system may switch from one "operating mode" to another. To define the dynamics of (9) completely, we will have to specify when these mode switches occur, what their effect will be on the state variables, and how a new mode will be selected. A proposal for answering these questions (cf. [10]) will be explained below. By the specification of the complete dynamics of (9) we describe a class of dynamical systems called linear complementarity systems.

Let $n$ denote the length of the vector $x(t)$ in the equations $(9 \mathrm{a}-9 \mathrm{~b})$ and let $k$ denote the number of inputs and outputs. There are then $2^{k}$ possible choices for the active index set. The equations of motion when the active index set is $I$ are given by

$$
\begin{aligned}
\dot{x}(t) & =A x(t)+B u(t) \\
y(t) & =C x(t)+D u(t) \\
y_{i}(t) & =0, \quad i \in I \\
u_{i}(t) & =0, \quad i \in I^{c} .
\end{aligned}
$$

We shall say that these equations represent the system in mode $I$. An equivalent and somewhat more explicit form is given by the (generalized) state equations

$$
\begin{aligned}
\dot{x}(t) & =A x(t)+B_{\bullet} u_{I}(t) \\
0 & =C_{I \bullet} x(t)+D_{I I} u_{I}(t)
\end{aligned}
$$

together with the output equations

$$
\begin{aligned}
& y_{I^{c}}(t)=C_{I^{c}} x(t)+D_{I^{c} I} u_{I}(t) \\
& u_{I^{c}}(t)=0 .
\end{aligned}
$$

At this point we need to digress in order to recall some facts concerning equations of the form (11), which can be derived from the geometric theory of linear systems (see $[23,1,13]$ for the general background). Denote by $V_{I}$ the consistent subspace 
of mode $I$, i. e. the set of initial conditions $x_{0}$ for which there exist smooth functions $x(\cdot)$ and $u_{I}(\cdot)$, with $x(0)=x_{0}$, such that (11) is satisfied. The space $V_{I}$ can be computed as the limit of the sequence defined by

$$
\begin{aligned}
V^{0} & =\mathbb{R}^{n} \\
V^{i+1} & =\left\{x \in V^{i} \mid \exists u \in \mathbb{R}^{|I|} \text { s.t. } A x+B_{\bullet_{I}} u \in V^{i}, C_{I_{\bullet}} x+D_{I I} u=0\right\} .
\end{aligned}
$$

There exists a linear mapping $F_{I}$ such that (11) will be satisfied for $x_{0} \in V_{I}$ by taking $u_{I}(t)=F_{I} x(t)$. The mapping $F_{I}$ is uniquely determined, and more generally the function $u_{I}(\cdot)$ that satisfies (11) for given $x_{0} \in V_{I}$ is uniquely determined, if the full-column-rank condition

$$
\operatorname{ker}\left[\begin{array}{c}
B_{\bullet I} \\
D_{I I}
\end{array}\right]=\{0\}
$$

holds and moreover we have

$$
V_{I} \cap T_{I}=\{0\},
$$

where $T_{I}$ is the subspace that can be computed as the limit of the following sequence:

$$
\begin{aligned}
T^{0} & =\{0\} \\
T^{i+1} & =\left\{x \in \mathbb{R}^{n} \mid \exists \tilde{x} \in T^{i}, \tilde{u} \in \mathbb{R}^{|I|} \text { s.t. } x=A \tilde{x}+B_{\bullet} \tilde{u}, C_{I \bullet} \tilde{x}+D_{I I} \tilde{u}=0\right\} .
\end{aligned}
$$

In the present context, the subspace $T_{I}$ is best thought of as the jump space associated to mode $I$, that is, as the space along which fast motions will occur that take an inconsistent initial state instantaneously to a point in the consistent space $V_{I}$; note that under the condition (15) this projection is uniquely determined. To make the interpretation of $T_{I}$ as a jump space precise, introduce the class of impulsive-smooth distributions that was studied by Hautus [8] (see also $[9,7]$ ). The general form of an impulsive-smooth distribution $\phi$ is

$$
\phi=p\left(\frac{d}{d t}\right) \delta+f
$$

where $p(\cdot)$ is a polynomial, $\frac{d}{d t}$ denotes the distributional derivative, $\delta$ is the delta distribution with support at zero, and $f$ is a distribution that can be identified with the restriction to $(0, \infty)$ of some function in $C^{\infty}(\mathbb{R})$. The class of such distributions will be denoted by $C_{\text {imp }}$. For an element of $C_{\text {imp }}$ of the form (17), we write $\phi\left(0^{+}\right)$ for the limit value $\lim _{t \downarrow 0} f(t)$. Having introduced the class $C_{\text {imp }}$, we can replace the system of equations (11) by its distributional version

$$
\begin{aligned}
\frac{d}{d t} x & =A x+B_{\bullet I} u_{I}+x_{0} \delta \\
0 & =C_{I_{0}} x+D_{I I} u_{I}
\end{aligned}
$$


in which the initial condition $x_{0}$ appears explicitly, and we can look for a solution of (18) in the class of vector-valued impulsive-smooth distributions. It was shown in [9] that if the conditions (14) and (15) are satisfied, then there exists a unique solution $\left(x, u_{I}\right) \in C_{\mathrm{imp}}^{n+|I|}$ to $(18)$ for each $x_{0} \in V_{I}+T_{I}$; moreover, the solution is such that $x\left(0^{+}\right)$is equal to $P_{V_{I}}^{T_{I}} x_{0}$, the projection of $x_{0}$ onto $V_{I}$ along $T_{I}$. The solution is most easily written down in terms of its Laplace transform:

$$
\begin{aligned}
\hat{x}(s) & =(s I-A)^{-1} x_{0}+(s I-A)^{-1} B_{\bullet} \hat{u}_{I}(s) \\
\hat{u}_{I}(s) & =-G_{I I}^{-1}(s) C_{I \bullet}(s I-A)^{-1} x_{0},
\end{aligned}
$$

where

$$
G_{I I}(s):=C_{I \bullet}(s I-A)^{-1} B_{\bullet I}+D_{I I}
$$

Note that the notation is consistent in the sense that $G_{I I}(s)$ can also be viewed as the $(I, I)$-submatrix of the transfer matrix $G(s):=C(s I-A)^{-1} B+D$. It is shown in [9] (see also [15]) that the transfer matrix $G_{I I}(s)$ associated to the system parameters in (11) is left invertible when (14) and (15) are satisfied. Since the transfer matrices $G_{I I}(s)$ that we consider are square, left invertibility is enough to imply invertibility, and so (by duality) we also have $V_{I}+T_{I}=\mathbb{R}^{n}$. Summarizing, we can list the following equivalent conditions. In the formulation of the theorem, we call a matrix $M$ over a field $\mathbb{F}$ totally invertible if none of its principal minors vanishes.

Theorem 3.1 Consider a time-invariant linear system with $k$ inputs and $k$ outputs, given by standard state space parameters $(A, B, C, D)$. The following conditions are equivalent.

1. For each index set $I \subset \bar{k}$, the associated system (11) admits for each $x_{0} \in V_{I}$ a unique smooth solution $(x, u)$ such that $x(0)=x_{0}$.

2. For each index set $I \subset \bar{k}$, the associated distributional system (18) admits for each initial condition $x_{0}$ a unique impulsive-smooth solution $(x, u)$.

3. The conditions (14) and (15) are satisfied for all $I \subset \bar{k}$.

4. The transfer matrix $G(s)=C(s I-A)^{-1} B+D$ is totally invertible (as a matrix over the field of rational functions).

In this paper we shall be interested in conditions for existence and uniqueness of solutions, and so we shall usually consider systems $(A, B, C, D)$ for which the above conditions hold.

After this digression, we can continue to define a notion of solution for the system (9). The following preliminary definitions will be needed. 
Definition 3.2 An impulsive-smooth distribution $\phi=p\left(\frac{d}{d t}\right) \delta+f$ as in (17) will be called initially nonnegative if the leading coefficient of the polynomial $p(\cdot)$ is positive, or, in case $p=0$, the smooth function $f$ is nonnegative on an interval of the form $(0, \varepsilon)$ with $\varepsilon>0$. A vector-valued impulsive-smooth distribution will be called initially nonnegative if each of its components is initially nonnegative in the above sense.

Definition 3.3 A triple of vector-valued impulsive-smooth distributions $(u, x, y)$ will be called an initial solution to (9) with initial state $x_{0}$ and solution mode I if

1. the triple $(u, x, y)$ satisfies the distributional equations

$$
\begin{aligned}
\frac{d}{d t} x & =A x+B u+x_{0} \delta \\
y & =C x+D u
\end{aligned}
$$

2. both $u$ and $y$ are initially nonnegative

3. $y_{i}=0$ for all $i \in I$ and $u_{i}=0$ for all $i \notin I$.

Given a vector $x_{0}$, we can consider the collection of all index sets $I$ such that there exists an initial solution to (9) with initial state $x_{0}$ and solution mode $I$. This collection will be denoted by $\mathcal{S}\left(A, B, C, D ; x_{0}\right)$, or simply by $\mathcal{S}\left(x_{0}\right)$ if the context is clear.

We are now ready to define the concept of a solution to (9). We shall first define what might be called a "full" solution to these equations, involving all of the ingredients that play a role. Depending on which aspects of the behavior one is interested in (for instance the continuous part or the switching part), one may then define related solution concepts which emphasize the aspects of interest.

Definition 3.4 Consider seven-tuples $\left(L, \tau, x_{e}, I, u_{c}, x_{c}, y_{c}\right)$ of the following form:

- $L$ is either $\{0, \ldots, N\}$ for some $N>0$ or $\mathbb{Z}_{+}$and is called the event label set

- $\tau$ is a monotonous function from $L$ to $\mathbb{R}_{+} \cup\{\infty\}$ whose values are called event times

- $x_{e}$ is a function from $L$ to $\mathbb{R}^{n}$ whose values are called event states

- $I$ is a function from $L$ to $2^{\bar{k}}$ whose values are called operating modes

- $u_{c}, x_{c}$, and $y_{c}$ are smooth functions defined on $[0, T) \backslash \tau(L)$, with values in $\mathbb{R}^{k}, \mathbb{R}^{n}$, and $\mathbb{R}^{k}$ respectively.

Such a seven-tuple is called a (full) solution to (9) on an interval $[0, T)$ if the following conditions hold:

1. $\tau(0)=0$ and $\sup _{i \in L} \tau(i)=T$ 
2. $I(i) \in \mathcal{S}\left(x_{e}(i)\right)$ for all $i \in L$

3. for all $i$ such that $i \in L, i+1 \in L$, and $\tau(i+1)=\tau(i)$, we have $x_{e}(i+1)=$ $P_{V_{I(i)}}^{T_{I(i)}} x_{e}(i)$

4. for all $i$ such that $i \in L, i+1 \in L$, and $\tau(i+1)>\tau(i)$, we have $x_{e}(i)=$ $\lim _{t \downarrow \tau(i)} x_{c}(t)$ and $x_{e}(i+1)=\lim _{t \uparrow \tau(i+1)} x_{c}(t)$

5. for all $i$ such that $i \in L$ and $i+1 \in L$, the triple $\left(u_{c}, x_{c}, y_{c}\right)$ satisfies (10) with $I=I(i)$ for $t \in(\tau(i), \tau(i+1))$

6. for all $t \in[0, T)$ we have $u_{c}(t) \geqslant 0$ and $y_{c}(t) \geqslant 0$.

The initial state corresponding to a given solution is the vector $x_{e}(0)$. The multiplicity of an event time $t \in \tau(L)$ is the number of labels $i \in L$ such that $\tau(i)=t$.

The above definition is more involved than would be necessary for the systems that we shall consider in this paper. Under conditions that will be formulated below, we have dominance of the continuous state in the sense that the evolution of the continuous state vector is completely determined by its initial value, and in particular does not depend on the choice of an initial mode. The definition as given is in a form that can be relatively easily modified to accommodate externally induced switching by redefining the set of continuation modes $\mathcal{S}(x)$.

Suppose now that we are mainly interested in the behavior of the continuous states of the system. To avoid trivial distinctions between solutions, we shall identify two piecewise continuous functions that are defined almost everywhere if they agree on their common domain of definition. A notion of solution that places emphasis on the continuous states can then be given as follows.

Definition 3.5 The continuous trace of a solution $\left(L, \tau, x_{e}, I, u_{c}, x_{c}, y_{c}\right)$ to (9) on an interval $[0, T)$ is the triple $\left(u_{c}, x_{c}, y_{c}\right)$ of vector-valued functions defined almost everywhere on $[0, T)$.

Definition 3.6 A triple $\left(u_{c}, x_{c}, y_{c}\right)$ of almost everywhere defined piecewise smooth vector-valued functions will be called a continuous-state solution to (9) if it is the continuous trace of a full solution.

The industrious reader may verify that, in the concrete case of the example of Section 2, the definitions above reproduce the rules that we formulated for that case.

\section{Well-posedness}

A basic issue concerns the existence and uniqueness of solutions. We understand well-posedness in the following "local" sense. 
Definition 4.1 The complementary-slackness system (9) is (locally) well-posed if for each initial state there exists an $\varepsilon>0$ such that (9) admits a unique continuousstate solution on $[0, \varepsilon)$.

The definition requires that for each initial vector in $\mathbb{R}^{n}$ there exists a unique solution on an interval of positive length starting with at most a finite number of jumps followed by smooth continuation. Note also that the definition expresses dominance of the continuous state, since no information about an initial discrete state ("starting mode") is required. Due to the possible occurrence of phenomena such as deadlock, ill-posedness is more common in discontinuous dynamical systems than it is in classical continuous systems. In particular, examples of linear complementarity systems that are not well-posed in the above sense are easy to find, see for instance [20].

To formulate sufficient conditions for well-posedness, we again need to introduce some linear systems terminology. The leading column indices $\eta_{1}, \ldots, \eta_{k}$ of a linear system $(A, B, C, D)$ with Markov parameters $G^{i}$ are defined by

$$
\eta_{j}:=\inf \left\{i \in \mathbb{N} \mid G_{\bullet j}^{i} \neq 0\right\}
$$

where we take $\inf \varnothing=\infty$. The leading row indices $\rho_{1}, \ldots, \rho_{k}$ of the system $(A, B, C, D)$ are defined by

$$
\rho_{j}:=\inf \left\{i \in \mathbb{N} \mid G_{j \bullet}^{i} \neq 0\right\} .
$$

For systems with totally invertible transfer matrices, the leading row and column indices are clearly all finite; in fact, we even have $\rho_{i} \leqslant n$ and $\eta_{i} \leqslant n$ for all $i$. The leading row coefficient matrix $M_{r}(A, B, C, D)$ and the leading column coefficient matrix $M_{c}(A, B, C, D)$ of a system $(A, B, C, D)$ with $k$ inputs and $k$ outputs are defined as follows:

$$
M_{r}(A, B, C, D):=\left(\begin{array}{c}
G_{1 \bullet}^{\rho_{1}} \\
\vdots \\
G_{k \bullet}^{\rho_{k}}
\end{array}\right), \quad M_{c}(A, B, C, D):=\left(G_{\bullet 1}^{\eta_{1}} \ldots G_{\bullet k}^{\eta_{k}}\right) .
$$

We also need some terminology from mathematical programming [4]: a square matrix $M \in \mathbb{R}^{k \times k}$ is said to be a $P$-matrix if all its principal minors are positive. Now we can state the following well-posedness result; see [10] for a proof.

Theorem 4.2 Let a linear complementarity system of the form (9) be given. Suppose that the system satisfies the conditions of Thm. 3.1, and that both the leading column coefficient matrix and the leading row coefficient matrix of the associated linear system $(A, B, C, D)$ are P-matrices. Under these conditions, the complementarity system is well-posed. Moreover, no solution requires a multiplicity of event times higher than two. 
The notion of well-posedness that is used here does not require continuous dependence on initial conditions. Actually, examples of linear complementarity systems in which the dependence on initial conditions is discontinuous are not hard to find (see [10]). For similar reasons of easy failure we have not included a claim on uniqueness of full solutions. Finally we have not claimed global existence of solutions since we have not discussed the possibility of event times having a finite accumulation point. Examples of complementarity systems that show a "Zeno behavior" do not seem to be easily found, so it may be conjectured that solutions will exist for all time under conditions similar to those of Thm. 4.2. Finally let us note that complementarity systems that are not well-posed in the forward sense may still be useful in the analysis of two-point boundary value problems, such as occur in the maximum principle for optimal control problems subject to unilateral state constraints.

\section{Conclusions}

In this paper we have discussed a class of hybrid systems that allow a relatively compact specification. Indeed, we can describe a system with $2^{k}$ discrete states essentially by giving the parameters of a linear system with $k$ inputs and $k$ outputs. Moreover, the analysis of the hybrid system can be carried out in terms of the associated linear system, which brings the results of linear multivariable systems theory to bear on a class of hybrid systems. The framework we presented is limited and needs to be extended in several directions, in particular to allow the inclusion of continuous and discrete inputs. In addition to the basic issues of well-posedness that have been discussed here, there is a need to explore for instance conditions for stability, efficient methods for simulation, compositional specification of large systems, and verification of safety properties by systematic search for worst-case situations.

\section{References}

[1] G. Basile and G. Marro. Controlled and Conditioned Invariants in Linear System Theory. Prentice Hall, Englewood Cliffs, NJ, 1992.

[2] R. W. Brockett. Hybrid models for motion control systems. In H. L. Trentelman and J. C. Willems, editors, Essays on Control. Perspectives in the Theory and its Applications (lectures and the mini-courses of the European control conference (ECC'93), Groningen, the Netherlands, June/July 1993), pages 29-53. Birkhäuser, 1993.

[3] D. W. Bushaw. Differential Equations with a Discontinuous Forcing Term. PhD thesis, Dept. of Math., Princeton Univ, 1952. 
[4] R. W. Cottle, J.-S. Pang, and R. E. Stone. The Linear Complementarity Problem. Academic Press, Boston, 1992.

[5] A. F. Filippov. Differential equations with discontinuous right-hand sides. Matematik. Sbornik., 51:99-128, 1960. In Russian. English translation: Am. Math. Soc. Transl. 62 (1964).

[6] P. A. Fuhrmann. Linear Systems and Operators in Hilbert Space. McGrawHill, New York, NY, 1981.

[7] A. H. W. Geerts and J. M. Schumacher. Impulsive-smooth behavior in multimode systems. Part I: State-space and polynomial representations. Automatica, 32:747-758, 1996.

[8] M. L. J. Hautus. The formal Laplace transform for smooth linear systems. In G. Marchesini and S.K. Mitter, editors, Mathematical Systems Theory, Lect. Notes Econ. Math. Syst. 131, pages 29-47. Springer, New York, 1976.

[9] M. L. J. Hautus and L. M. Silverman. System structure and singular control. Lin. Alg. Appl., 50:369-402, 1983.

[10] W. P. M. H. Heemels, J. M. Schumacher, and S. Weiland. Linear complementarity systems. In preparation.

[11] T. Kailath. Linear Systems. Prentice-Hall, Englewood Cliffs, N.J., 1980.

[12] C. W. Kilmister and J. E. Reeve. Rational Mechanics. Longmans, London, 1966.

[13] M. Kuijper. First-Order Representations of Linear Systems. Birkhäuser, Boston, 1994.

[14] J. P. LaSalle. Time optimal control systems. Proc. Natl. Acad. Sci. U.S., 45:573-577, 1959.

[15] A. S. Morse and W. M. Wonham. Status of noninteracting control. IEEE Trans. Automat. Contr., AC-16:568-581, 1971.

[16] H. Nijmeijer and A. J. van der Schaft. Nonlinear Dynamical Control Systems. Springer-Verlag, Berlin, 1990.

[17] J. Pérès. Mécanique Générale. Masson \& Cie., Paris, 1953.

[18] L. S. Pontryagin, V. G. Boltyanskii, R. V. Gamkrelidze, and E. F. Mishchenko. The Mathematical Theory of Optimal Processes. Interscience, New York, 1962.

[19] A. J. van der Schaft and J. M. Schumacher. Complementarity modeling of hybrid systems. Report BS-R9611, CWI, Amsterdam, 1996.

URL: http://www. cwi.nl/ftp/CWIreports/BS/BS-R9611.ps.Z. 
[20] A. J. van der Schaft and J. M. Schumacher. The complementary-slackness class of hybrid systems. Math. Contr. Signals Syst., 9:266-301, 1996.

[21] L. Vandenberghe, B. L. De Moor, and J. Vandewalle. The generalized linear complementarity problem applied to the complete analysis of resistive piecewise-linear circuits. IEEE Trans. Circuits Syst., CAS-36:1382-1391, 1989.

[22] H. S. Witsenhausen. A class of hybrid-state continuous-time dynamic systems. IEEE Transactions on Automatic Control, AC-11:161-167, 1966.

[23] W. M. Wonham. Linear Multivariable Control: A Geometric Approach. Springer Verlag, New York, 3rd edition, 1985.

J.M. Schumacher

CWI

P.O. Box 94079

1090 GB Amsterdam

The Netherlands

Tel. +31-20-5924090

Fax +31-20-5924199

E-mail Hans.Schumacher@cwi.nl 